\title{
Study of period number effect in the superlattice infrared photodetector
}

\author{
J.H. Lu *, Y.Y. Yang, C.C. Chen, C.H. Kuan, H.T. Chen, S.C. Lee \\ Department of Electrical Engineering and Graduate Institute of Electronics Engineering, National Taiwan University, \\ Taipei 10617, Taiwan, ROC
}

\begin{abstract}
Superlattices have been demonstrated previously by our group in the design of the multicolor infrared photodetector. In general, the period number of the superlattice may be up to several dozens. In this paper, we have investigated the performance of the infrared photodetectors especially with 3, 5 and 15 periods. The detector structure contains a thick blocking barrier embedded between two superlattices with different period numbers but with the same well and barrier widths. This double-superlattice structure shows switchable spectral responses between two spectral regions by the voltage polarities. The photoresponse in each spectral region is also tunable by the magnitude of the applied voltage. The voltage-dependent behavior reveals the photoelectron relaxation and transport mechanism in the superlattice miniband. Superlattice with few periods has high electron group velocity, less relaxation effect and less collection efficiency. Therefore the superlattice with few periods may have better responsivity and narrower photoresponse range than the one with many periods. Based on the experimental results of our devices, it is observed that the superlattice with fewer periods has better detectivity, responsivity, wider range of the operational temperature, and more flexible miniband engineering than the conventional multiple quantum well infrared photodetector.
\end{abstract}

(C) 2003 Elsevier B.V. All rights reserved.

Keywords: Infrared photodetector; Intersubband; Quantum well infrared photodetector; Superlattice

\section{Introduction}

Quantum well infrared photodetectors (QWIPs), especially operating in 3-5 and 8-12 $\mu \mathrm{m}$ wavelength ranges, have been widely investigated over the past decades and are required for many applications, such as thermal imaging and remote temperature sensing. Among them, GaAs/AlGaAs QWIPs have attracted much attention because of

\footnotetext{
${ }^{*}$ Corresponding author.

E-mail addresses: jeter@hurryman.ee.ntu.edu.tw (J.H. Lu), kuan@cc.ee.ntu.edu.tw (C.H. Kuan).
}

their characteristics, such as high uniformity, high sensitivity, high yield and low cost [1]. In addition to $\mathrm{QW}$, the superlattice is another promising structure for an infrared photodetector and has drawn much attention. The superlattice intersubband photodetector with a graded barrier was fabricated for photovoltaic detection in the range of 3.6-6.3 and 8-10.5 $\mu \mathrm{m}$ in 1988 [2] and 1990 [3], respectively. The superlattice alone was applied in the detection of the wavelength range of 5-10 $\mu \mathrm{m}$ in 1991 [4]. The superlattice with a blocking layer operated in a low bias region was demonstrated $[5,6]$. The works on the superlattice infrared photodetectors (SLIPs) indicate that the advantages 
of the superlattice-based infrared photodetector include a broader absorption spectrum, lower operation voltage and more flexible miniband engineering than the conventional QWIP.

Although the absorption between the minibands in the superlattice increases with the period number, only the photoexcited electrons in few quantum wells near the contact layer contribute to the photocurrent as a result of the relaxation and scattering mechanisms. The relaxation mechanisms of photoelectrons in the second miniband include interminiband and intraminiband relaxations $[7,8]$. The intraminiband relaxation makes the electrons to accumulate in the bottom state of the second miniband, and therefore limits the short wavelength responsivity. The effect of relaxation is more remarkable with longer transit distance of photoelectrons. Hence we expect that the short wavelength responsivity will be apparent for fewperiod SLIPs.

In this paper, we will compare the electrical and optical properties of the sample with 3-period and 5-period $\mathrm{GaAs} / \mathrm{Al}_{0.28} \mathrm{Ga}_{0.72} \mathrm{As}$ superlattices with another sample containing 3-period and 15-period $\mathrm{GaAs} / \mathrm{Al}_{0.28} \mathrm{Ga}_{0.72} \mathrm{As}$ superlattices. In addition to the superlattices, the samples also contain current blocking layers. Because the resistance of SL structure is very low, the applied bias almost completely drops across the blocking layer and varies its energy shape at the same time. Therefore, the current blocking layer not only reduces the dark current but also acts as a voltage-tuned highpass energy filter for the photoexcited carriers. Hence the spectral responsivity is tunable by external bias and the multiwavelength operation is enabled for temperature sensing.

\section{Design principle}

The sample design is based on the consideration of three factors. They are the difference between QWIP and SLIP, the relationship of the electron group velocity and the superlattice period number, and relaxation mechanism in SLIP.

The intersubband photodetectors constructed from multiple quantum wells or superlattices are shown in Fig. 1. Infrared detection is via the

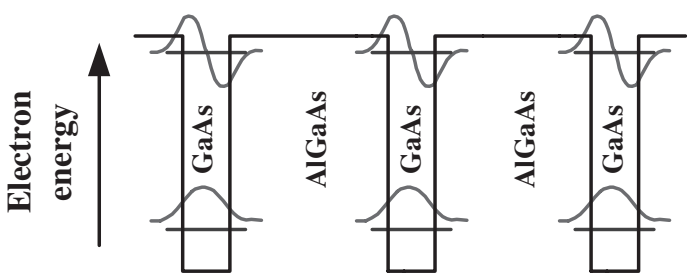

Distant quantum wells

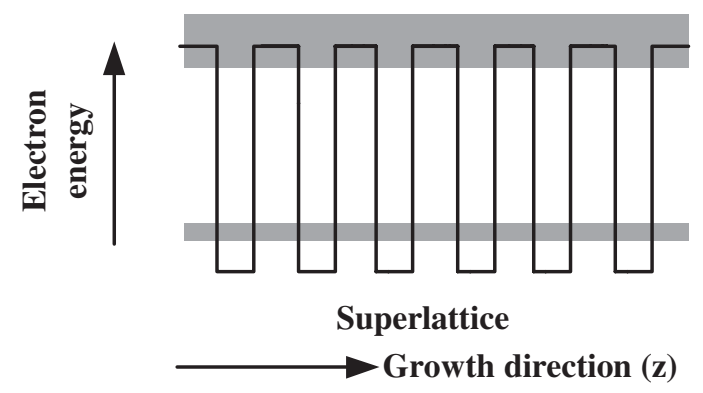

Fig. 1. Schematic drawing of the quantum well and superlattice structures.

intersubband transitions within the multiple quantum wells or superlattices. Their difference is that the adjacent wells are separated by thick barriers in QWIP but thin barriers in SLIP. Hence the coupling of electron wavefunctions in adjacent wells is negligible in the quantum wells, and the electrons are quantized into discrete energy states. For SLIP, electron wavefunctions in adjacent wells are coupled to form the minibands in the superlattice region. The minibands instead of discrete states make the superlattice to have broader spectral response than the quantum well. In SLIP, the photoelectrons in the second miniband are supplied by those of the first miniband, conversely in QWIP the electrons can be only supplied by those overcoming the barriers in adjacent wells. Based on this mechanism, it is believed that the SLIP with more periods is better since higher absorption and responsivity are expected.

Since no voltage is dropped on superlattice, the transport of the photoelectrons through the second miniband is in terms of the group velocity instead of the drift velocity. Fig. 2 is the plot of the energy dispersion for $E$ versus $k$ of the second miniband for the superlattice with infinite periods and the same well and barrier structures we use in 


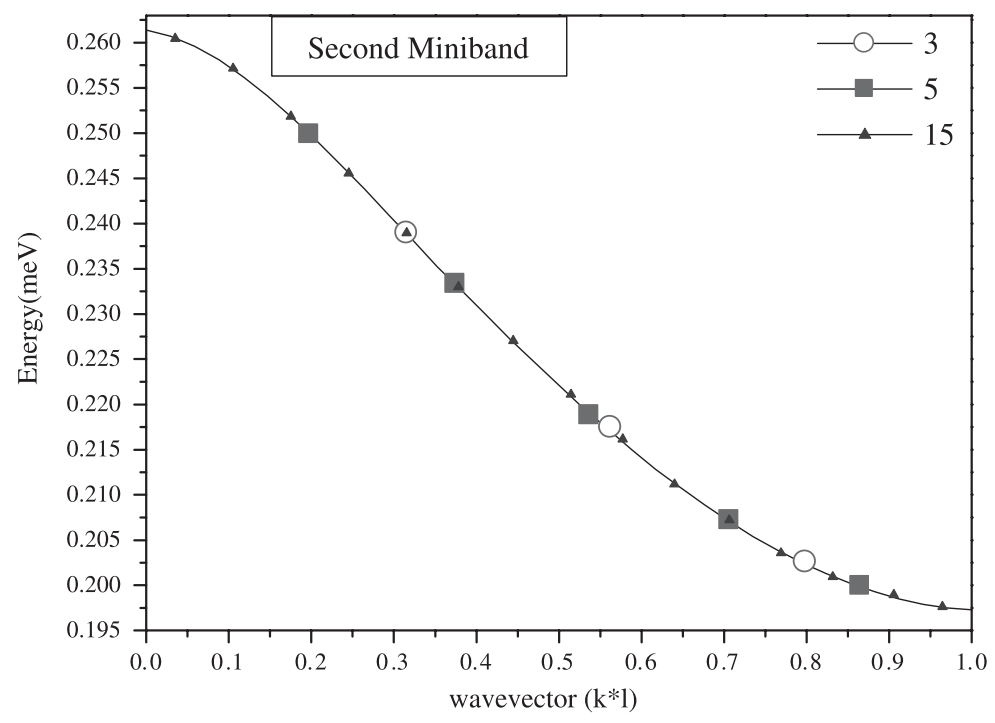

Fig. 2. The miniband dispersions of the second miniband for 3-, 5-, and 15-, and infinite-period SL's. According to $\vec{v}_{k}=(1 / \hbar) \nabla_{k} \vec{E}$, the photoelectrons at the bottom and top states of the second miniband in 3-period SLIP have higher velocity than in 15-period miniband.

the experiment. The discrete energies for the superlattice with the period numbers of 3,5 , and 15 are also marked in the same plot. It is noted that the discrete energy states for the finite superlattice are close to the middle of the miniband for the infinite one. According to the group velocity $\left(\overrightarrow{v_{k}}=(1 / \hbar) \nabla_{k} \vec{E}\right)$, the photoelectrons at the top and the bottom states of the second miniband have slower velocity in 15-period superlattice than that in 3-period one. In the same way, the electron's group velocity is close to zero at the bottom state of the second miniband for the superlattice with many periods. Therefore, SLIP with few periods will have better performance based on the consideration of the group velocity.

For SLIP with many periods, the transit distance of photoelectrons is relative long. Because of the longer distance, photoelectrons will suffer more scattering and relaxation processes into the first miniband or the bottom states in the second miniband. This may result in low collection efficiency. To reduce the relaxation effect, SLIP with few periods is preferred.

Based on the above three factors, we find the period number of SLIP is a trade-off factor. SLIP with many periods will have better supplying mechanism but the one with few periods will have faster group velocity and less relaxation effect. In our experiments, we choose 3-, 5- and 15-period superlattices as our investigated targets.

\section{Sample structure}

Two samples are utilized in this paper. Sample A has 3-period and 5-period superlattices, and sample B has 3-period and 15-period superlattices. In our experiments, samples are grown on a semiinsulating GaAs substrate by molecular beam epitaxy (MBE). Both samples contain $5000 \AA$ heavily doped $\left(n=1 \times 10^{18} \mathrm{~cm}^{-3}\right)$ GaAs bottom contact, a bottom superlattice, a $2000 \AA$ $\mathrm{Al}_{0.28} \mathrm{Ga}_{0.72} \mathrm{As}$ undoped current blocking layer, a top superlattice and a $5000 \AA$ heavily doped $\left(n=1 \times 10^{18} \mathrm{~cm}^{-3}\right)$ GaAs top contact layer. Each period of the superlattice consists of $6 \mathrm{~nm}$ GaAs well and $4 \mathrm{~nm}$ undoped $\mathrm{Al}_{0.28} \mathrm{Ga}_{0.72} \mathrm{As}$ barrier, but the well is doped with $5 \times 10^{17} \mathrm{~cm}^{-3}$ of $\mathrm{Si}$ in sample A and $1 \times 10^{18} \mathrm{~cm}^{-3}$ in sample B. The conduction band offset of $\mathrm{Al}_{x} \mathrm{Ga}_{1-x}$ As relative to $\mathrm{GaAs}$ is taken as $810 x \mathrm{meV}$ [9]. Table 1 shows the comparison and the differences between samples $\mathrm{A}$ and $\mathrm{B}$. 
Table 1

Comparison of samples A and B

\begin{tabular}{lll}
\hline & Sample A & Sample B \\
\hline Front $S L$ & & \\
Period number & 5 & 15 \\
First miniband range & $55.4-64.7 \mathrm{meV}$ & $54.9-65.4 \mathrm{meV}$ \\
Second miniband range & $199.7-251.7 \mathrm{meV}$ & $197.6-260.4 \mathrm{meV}$ \\
Absorption wavelength & $6.32-9.19 \mu \mathrm{m}$ & $6.03-9.38 \mu \mathrm{m}$ \\
Rear $S L$ & & \\
Period number & 3 & 3 \\
First miniband range & $56.2-63.8 \mathrm{meV}$ & $56.2-63.8 \mathrm{meV}$ \\
Second miniband range & $202.3-239 \mathrm{meV}$ & $202.3-239 \mathrm{meV}^{-19}$ \\
Absorption wavelength & $6.78-9.0 \mu \mathrm{m}$ & $6.78-9.0 \mu \mathrm{m}$ \\
Doping level in GaAs well & $5 \times 10^{17} / \mathrm{cm}^{3}$ & $1 \times 10^{18} / \mathrm{cm}^{3}$ \\
Peak detectivity $D^{*}$ at $20 \mathrm{~K}$ under positive bias & $3.7 \times 10^{10} \mathrm{~cm} \mathrm{~Hz}^{0.5} / \mathrm{W}$ & $9.33 \times 10^{10} \mathrm{~cm} \mathrm{~Hz}^{0.5} / \mathrm{W}$ \\
Peak detectivity $D^{*}$ at $20 \mathrm{~K}$ under negative bias & under $1 \mathrm{~V}, 9 \mu \mathrm{m}$ & under $1.7 \mathrm{~V}, 9.4 \mu \mathrm{m}$ \\
& $2.35 \times 10^{10} \mathrm{~cm} \mathrm{~Hz}^{0.5} / \mathrm{W}$ & $4.96 \times 10^{9} \mathrm{~cm} \mathrm{~Hz}^{0.5} / \mathrm{W}$ \\
\hline
\end{tabular}

All energy levels are relative to GaAs conduction band.

The energy height of the blocking layer is about $227 \mathrm{meV}$ and a little bit higher than the bottom state of the second miniband of the superlattices. The barrier can act as a high-pass energy filter for the excited photoelectrons, and also make the response tunable by external bias. At around zero bias, photoelectrons with energy higher than the energy filter can pass through it, while those with energy lower are blocked. At high biases, because of the assistance of the strong electric field on the blocking barrier, part of the blocked photoelectrons can tunnel through the current blocking barrier and contribute to the photocurrent. As a result, the energy filter can be used to distinguish the energy of the photoelectron and resolve the photoelectron distribution versus energy.

The sample was processed into a $100 \times 100 \mu \mathrm{m}^{2}$ mesa by using standard photolithography, chemical wet etching, evaporation and lift-off processes. The top and bottom contacts were made by evaporating $60 \mathrm{~nm} \mathrm{Ni} / \mathrm{Ge} / \mathrm{Au}$ and $240 \mathrm{~nm} \mathrm{Au}$. After evaporation and lift-off, thermal annealing at $390{ }^{\circ} \mathrm{C}$ for $24 \mathrm{~s}$ was used to make ohmic contacts with the $\mathrm{n}^{+}$contact layers in the semiconductor. Finally, a $45^{\circ}$ facet on the substrate was made to allow the detector to probe the TM polarized infrared radiation.

\section{Results and discussion}

\subsection{Current-voltage characteristics}

The $I-V$ curves of samples A and B are shown in Fig. 3(a) and (b), respectively. The solid curves are the dark current measured without illumination by infrared radiation at temperature $20-100$ $\mathrm{K}$, and the dashed curve is the photocurrent at $12.5 \mathrm{~K}$ under the illumination of the room-temperature $(\sim 300 \mathrm{~K})$ background. Positive bias means the top contact is biased positively while the bottom contact is at ground potential. From Fig. 3 , it is expected that the asymmetrical current characteristic with the voltage polarity is observed. The cause is that under different polarity of bias, a different SLIP will be operated. The operational mechanisms under positive bias are shown in Fig. 4. The photocurrent corresponds to the photoelectrons injected from the second miniband in the bottom superlattice. Those photoelectrons leave a positive charge to attract electrons from bottom contact and therefore the photocurrent is formed in the external circuit. On the other hand, the photoelectrons in the top superlattice attract those from the top contact. This is an internal current circulation that cannot be measured by external circuit. It is then concluded that the photocurrent 

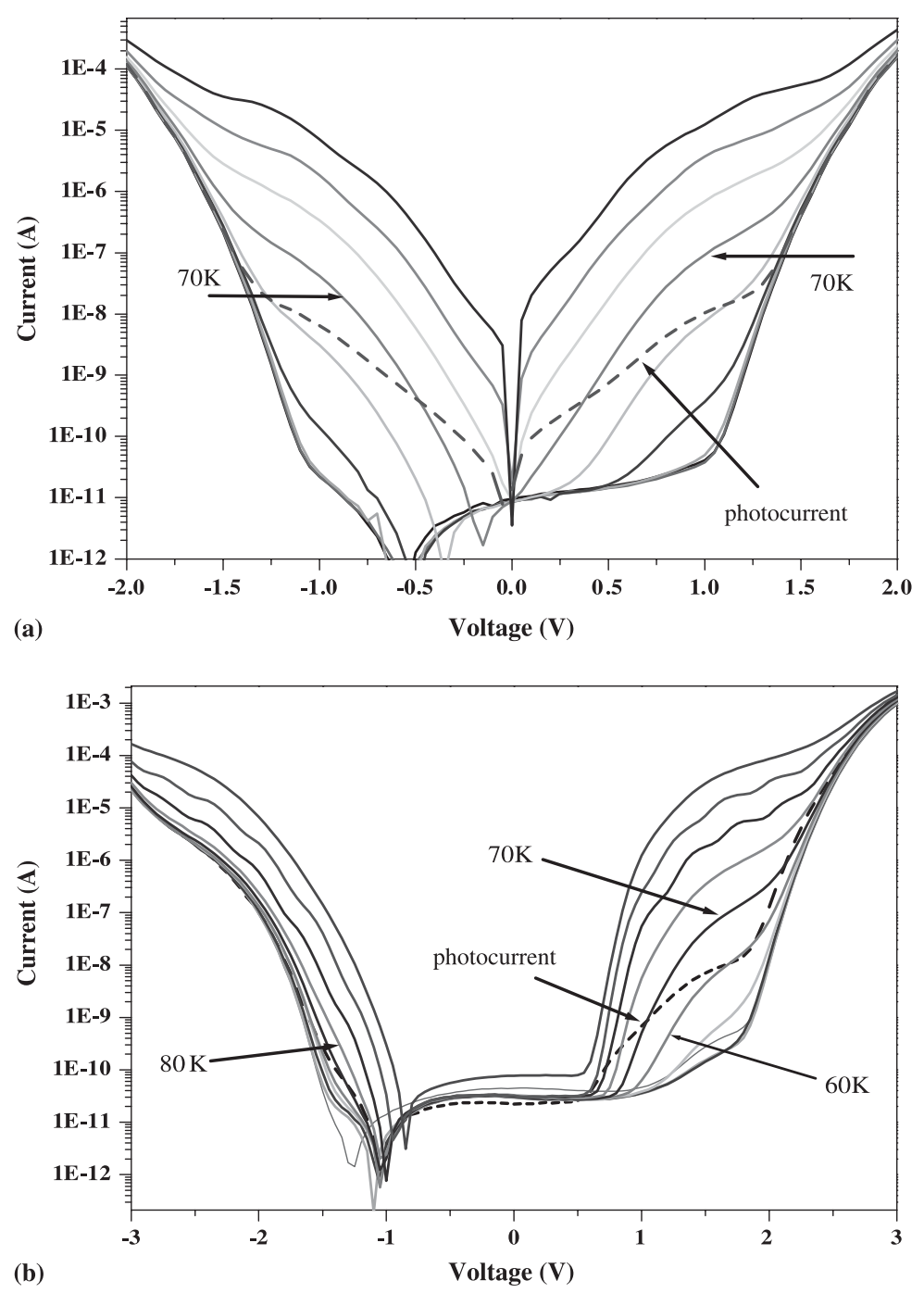

Fig. 3. The current-voltage characteristics of (a) sample A and (b) sample B. The solid lines are the dark-current $I-V$ curves from 20 to $100 \mathrm{~K}$. The dash line is the photocurrent at $20 \mathrm{~K}$ with the illumination of the room temperature background.

under positive bias is attributed to the bottom superlattice and under negative bias is attributed to the top superlattice.

In Fig. 3(a), by comparison between the dashed and solid curves, it is observed that the temperature with background limited performance for sample $\mathrm{A}$ is $70 \mathrm{~K}$ when positive bias $<0.38 \mathrm{~V}$ and negative bias $<0.46 \mathrm{~V}$. Because the period number in each superlattice in sample $\mathrm{A}$ is not so quite different, the $I-V$ characteristics under positive and negative bias are quite similar if neglecting the current with the magnitude less than $10^{-10} \mathrm{~A}$ due to the limitation of the current instrument. For sample $\mathrm{B}$, the temperature for the background limited performance is up to $70 \mathrm{~K}$ when positive bias $<1 \mathrm{~V}$ and negative bias $<1.3 \mathrm{~V}$. The period numbers of each superlattice in sample $B$ differ quite a lot, and the resulted difference of current magnitude under positive and negative is more obvious. Based on the consideration of the background limited performance, it is found that the SLIP with a fewer-period number has a compatible 


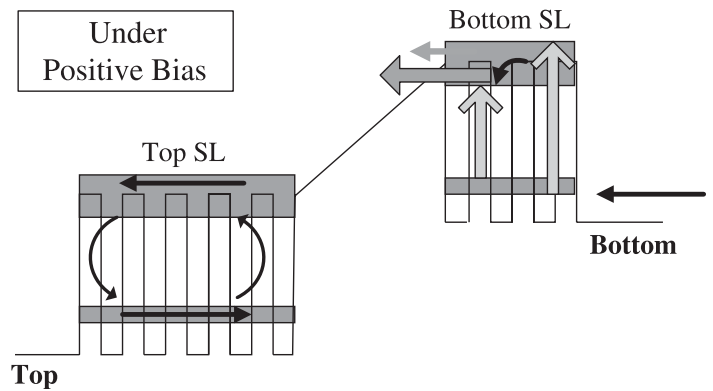

Fig. 4. Schematic illustration of operational mechanisms under positive bias. Only bottom SL is active under positive bias. result, although its absorption coefficient is expected to be lower.

\subsection{Responsivity and detectivity}

\subsubsection{Sample A (SLIP with 3-period and 5-period)}

Fig. 5 is the comparison of spectral response of sample A under positive and negative bias. In order to identify the relationship between spectrum and bias voltage, we plot spectra with high bias and low bias separately. From Fig. 5, the photoresponse peaks range from 6.7 to $7.4 \mu \mathrm{m}$

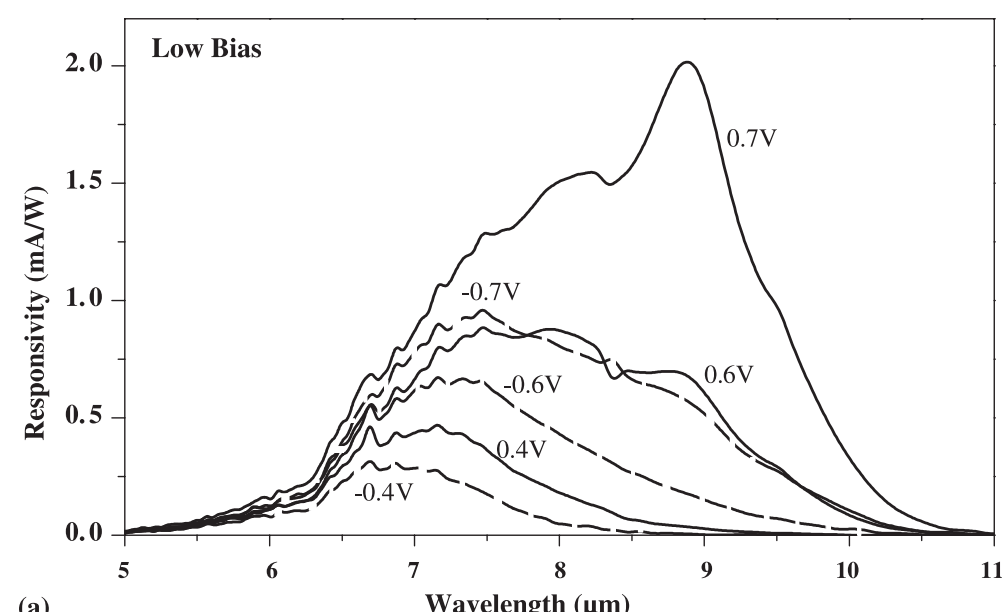

(a)

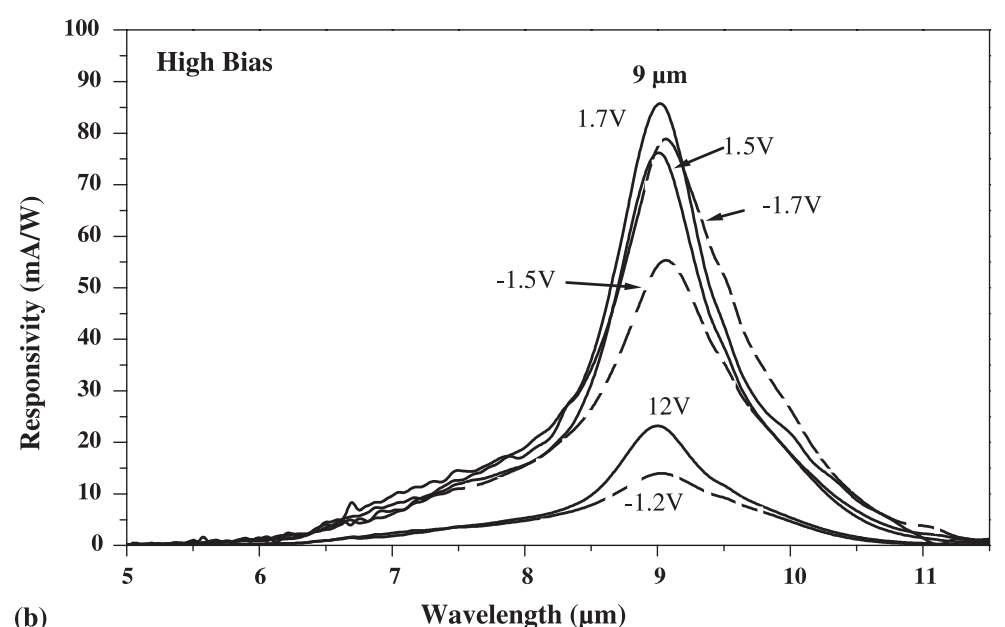

Fig. 5. The comparison of responsivity of sample A under positive and negative biases at (a) low biases and (b) high biases. The solid lines are responsivity under positive biases and dashed lines are the responsivity under negative biases. The $9 \mu \mathrm{m}$ responsivity is suppressed at low bias, while it increases with the applied voltage increasing. 
under applied voltage $0.4-0.6 \mathrm{~V}$. These relative short wavelength responses are mainly due to the high-energy photoelectron above the barrier height of the blocking layer. With increasing voltage, the long wavelength response dominates the spectrum. It is attributed that the photoelectrons in the lowest state of second miniband can tunnel through the barrier with the assistance of the strong electric field at high biases. For the applied voltage $>1.0 \mathrm{~V}$, the spectrum has a peak at about $9.0 \mu \mathrm{m}$ and the line shape is insensitive to bias voltage. In the same way, the spectral response under negative bias, i.e. corresponding to the top superlattice, ranges from 6.7 to $7.5 \mu \mathrm{m}$ under the low bias of -0.4 to $-0.7 \mathrm{~V}$ and has a long wavelength peak at $9 \mu \mathrm{m}$ under negative bias $>0.9 \mathrm{~V}$. It is obvious that the photoresponse of the two superlattices can be tuned by the bias magnitude due to the energy filter effect of the blocking layer.

It is observed from Fig. 5 that no matter under low or high bias, 3-period SLIP has higher responsivity than 5-period SLIP. Because of the wider miniband range, the photoresponse of 5period superlattice is a little bit broader than the one of 3-period superlattice. This characteristic is more obvious especially under high bias.

The photoresponse of 3-period and 5-period SLIP in sample A under $1.3 \mathrm{~V}$ at 20 and $100 \mathrm{~K}$ is shown in Fig. 6(a). It is observed that both 3-period and 5-period SLIP can work well until $100 \mathrm{~K}$ and have advantages of little temperature dependence of the spectral responsivity.

Suppose the noise performance is shot noise. The associated detectivity can be calculated with the responsivity and the dark current. The peak detectivity $D^{*}$ at $20 \mathrm{~K}$ is $3.7 \times 10^{10} \mathrm{~cm} \mathrm{~Hz}^{0.5} / \mathrm{W}$ under $1 \mathrm{~V}$ and $9 \mu \mathrm{m}$, and $2.35 \times 10^{10} \mathrm{~cm} \mathrm{~Hz}^{0.5} / \mathrm{W}$ at $9 \mu \mathrm{m}$ under $-1 \mathrm{~V}$. This result also shows that 3period SLIP has a better detectivity than 5-period SLIP. Because the temperature of background limited performance is $70 \mathrm{~K}$ for sample A, we also estimate the value of detectivity at $80 \mathrm{~K}$. The $D^{*}$ at $80 \mathrm{~K}$ is $7.16 \times 10^{8} \mathrm{~cm} \mathrm{~Hz}^{0.5} / \mathrm{W}$ under $1.3 \mathrm{~V}$ and $6.53 \times 10^{8} \mathrm{~cm} \mathrm{~Hz}^{0.5} / \mathrm{W}$ under $-1.3 \mathrm{~V}$. Because of the rapid increasing of dark current at high temperature, the detectivity decreases as the temperature is raised.
From Fig. 2, the group velocity in the second miniband has a maximum value in the intermediate zone of the miniband. The fewer the period number of superlattice is, the faster the electrons group velocity is. Therefore the photoelectrons in few periods SLIP has higher group velocity to pass through the barrier. Although the absorption coefficient may be lower in 3-period superlattice than that in 5-period one, electrons in 3-period superlattice have higher group velocity and less accumulation in the bottom state of the second miniband than in 5-period one. Based on the experimental results of sample A, SLIP with few periods indeed has the better responsivity and detectivity.

\subsubsection{Sample B (SLIP with 3-period and 15- period)}

Fig. 7 shows the spectral response of sample B under positive and negative bias. The solid lines are the photoresponses under positive bias for the 3-period superlattice and dashed lines are the ones under negative bias for the 15-period superlattice. The response is dominated by short-wavelength transition under low bias and shift to long wavelength as voltage increasing just like sample A. Under positive bias, the main peak is at $6.7 \mu \mathrm{m}$ when bias $<0.9 \mathrm{~V}$ and the peak at $7.8 \mu \mathrm{m}$ appears at higher bias. For bias $>1.3 \mathrm{~V}$, the main peak is at $9.35 \mu \mathrm{m}$ and the line shape of the responsivity does not vary with the bias voltage anymore. For negative bias, the main peak is $6.65 \mu \mathrm{m}$ at low bias and is $9.5 \mu \mathrm{m}$ under high bias. The effect of the blocking barrier, i.e. the voltage tunable spectrum, is also observed in sample B.

By comparison between solid and dashed lines, some characteristics are observed. Firstly, except under low bias, 3-period superlattice has the narrow photoresponse range while 15-period superlattice has the broader one. This result is consistent with the data shown in Table 1. Secondly, under 1.2 $\mathrm{V}$, three responsivity peaks such as $6.5,7.8$ and 9.1 $\mu \mathrm{m}$ can be distinguished clearly. These three peaks approximately correspond to three energy levels formed by 3-period superlattice. Thirdly, from Fig. 7(b) and (c), the superlattice with few period numbers still has a better responsivity for long wavelength response and for short wavelength 


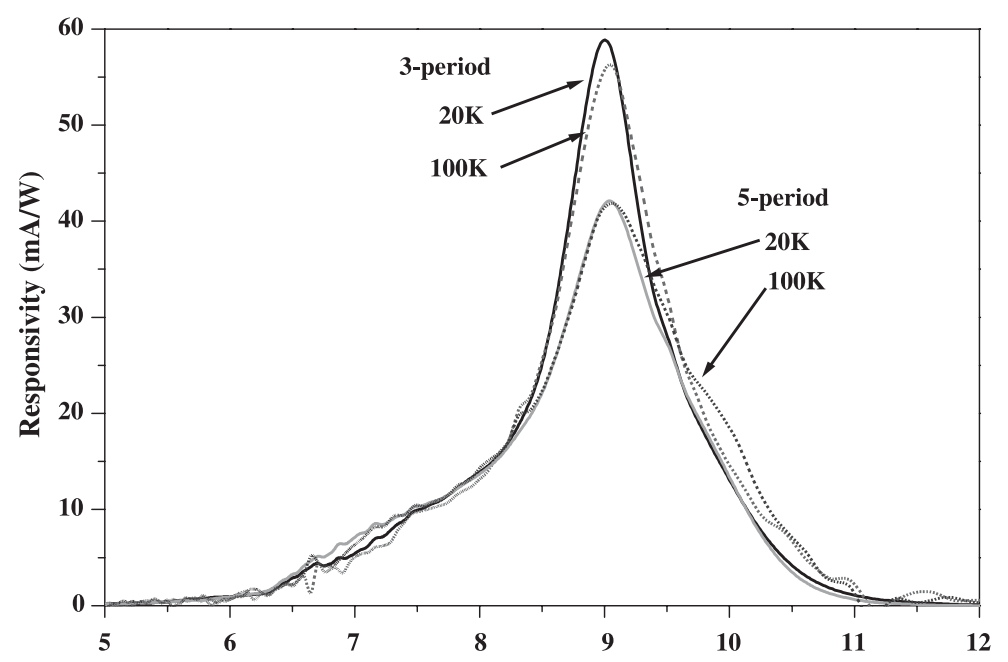

(a)

Wavelength $(\mu \mathrm{m})$

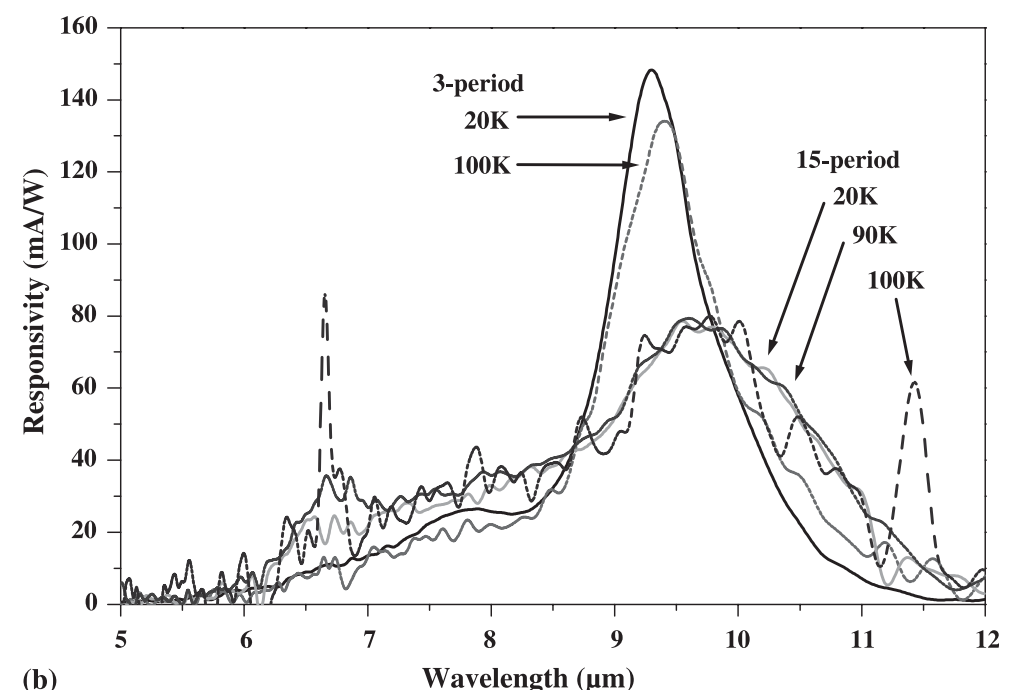

Fig. 6. (a) The spectral responsivity of 3-period and 5-period superlattice in sample A at $1.3 \mathrm{~V}$ at temperature 20 and $100 \mathrm{~K}$. (b) The spectral responsivity of sample B of 3-period superlattice at $1.8 \mathrm{~V}$ at temperature 20 and $100 \mathrm{~K}$ and 15 -period one at $-2.7 \mathrm{~V}$ at temperature 20, 90 and $100 \mathrm{~K}$.

response, the superlattice with many period numbers has a better performance.

Fig. 6(b) is the responsivity of the 3-period superlattice and 15-period superlattice in sample B at different temperature. For temperature operating range, the 3-period SLIP can work well till $100 \mathrm{~K}$. This result is consistent with sample A's. On the other hand, the highest operational temperature is $90 \mathrm{~K}$ for 15-period SLIP.
Assuming the shot noise behavior, the peak detectivity $D^{*}$ is $9.33 \times 10^{10} \mathrm{~cm} \mathrm{~Hz}^{0.5} / \mathrm{W}$ under $V=1.7$ $\mathrm{V}$ at wavelength $9.4 \mu \mathrm{m}$ and $4.96 \times 10^{9} \mathrm{~cm} \mathrm{~Hz}^{0.5} / \mathrm{W}$ for $V=-1.6 \mathrm{~V}$ at $6.7 \mu \mathrm{m}$. At $T=80 \mathrm{~K}$, the $D^{*}$ is $1.88 \times 10^{9} \mathrm{~cm} \mathrm{~Hz}^{0.5} / \mathrm{W}$ for $V=1.8 \mathrm{~V}$ at $9.4 \mu \mathrm{m}$ and $1.31 \times 10^{9} \mathrm{~cm} \mathrm{~Hz}^{0.5} / \mathrm{W}$ for $V=-1.6 \mathrm{~V}$ at $6.7 \mu \mathrm{m}$. It is observed that at $80 \mathrm{~K}, 15$-period superlattice detectivity at short wavelength is better and 3-period superlattice detectivity at long wavelength is better. 


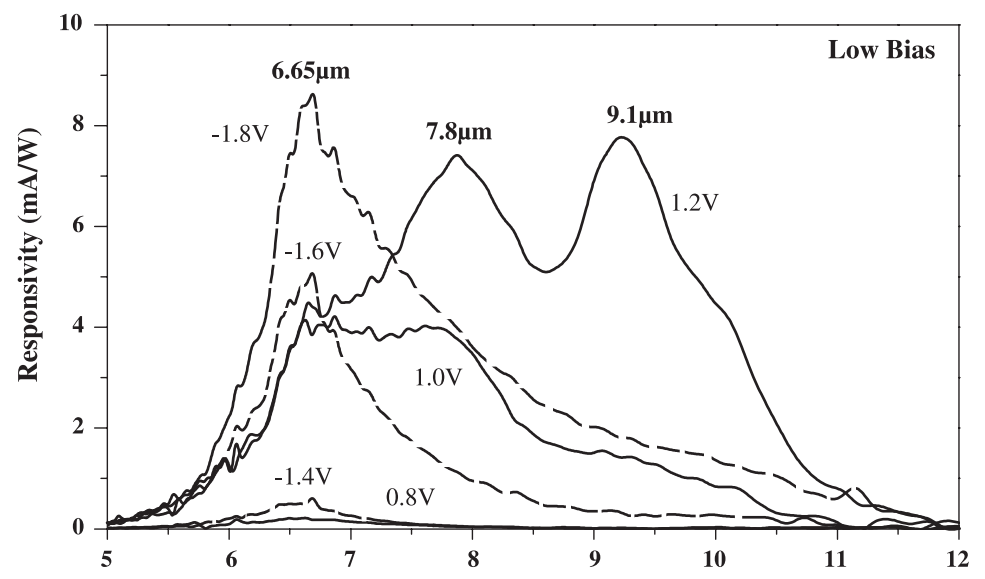

(a)

Wavelength $(\mu \mathrm{m})$

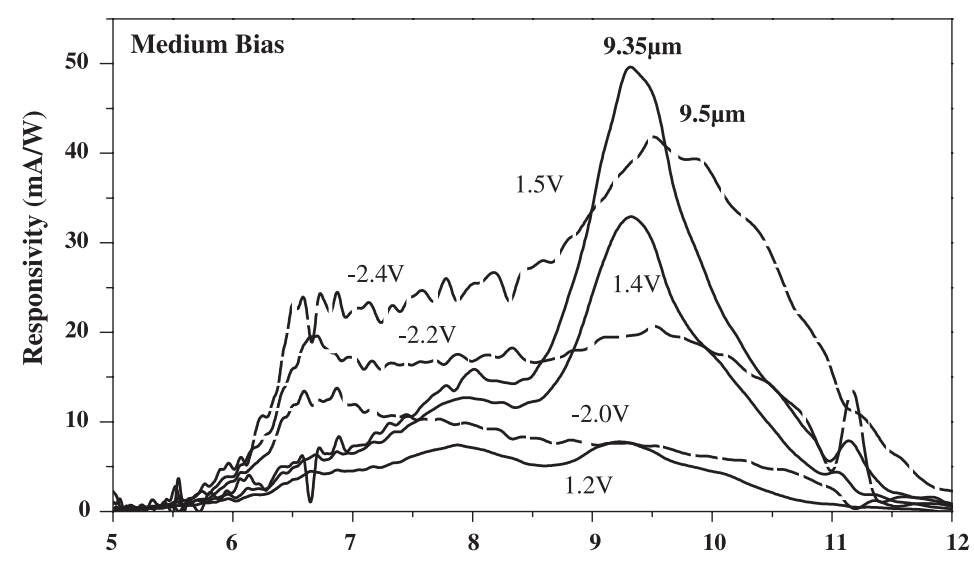

(b)

Wavelength $(\mu \mathrm{m})$

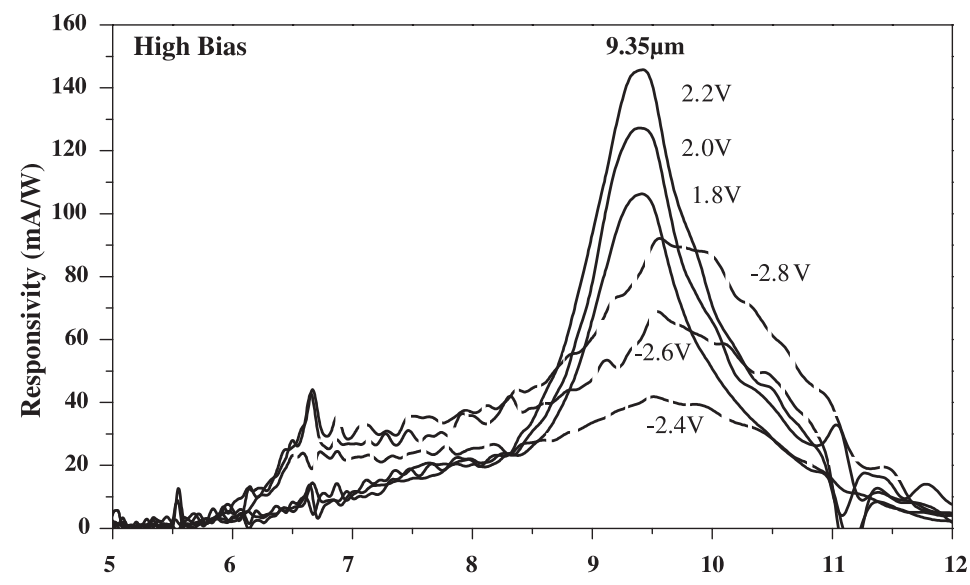

(c)

Wavelength $(\mu \mathrm{m})$

Fig. 7. The responsivities of sample B with (a) low bias magnitude, (b) medium bias magnitude and (c) high bias magnitude of both positive and negative biases. The solid lines are responsivity under positive biases and dashed lines are the responsivity under negative biases. The peak responsivity is shifted from short to long wavelength as biases increasing. 
It is well known that the intrasubband relaxation is due to electron scattering from the upper state to lower state by means of the interaction with a longitudinal optical (LO) phonon [10]. Because the energy spacing of the discrete level in the subband is less in 15-period SLIP than in 3-period SLIP, the intrasubband relaxation time in 15period SLIP is shorter than in 3-period SLIP. Moreover, the second miniband of 15-period SLIP has 15 discrete energy states and photoelectrons have more states to relax into. Hence many electrons will accumulate in the bottom state of the second miniband in 15-period superlattice. It is believed that the absorption coefficient is larger in 15-period superlattice than in 3-period one. Therefore more electrons excited by short wavelength will accumulate in the bottom state of the second miniband in 15-period superlattice than that in 3-period superlattice. Under high bias, because of the assistance of strong electric field, those accumulated electrons can tunnel through the blocking barrier and become the tunneling photocurrent. This is the cause of the responsivity at short wavelength range is higher for many periods superlattice than that for few periods one. On the other hand, electrons excited by long wavelength have only one possibility to relax into the first miniband. Therefore the accumulation is not the dominating factor for long wavelength response. For 15-period superlattice, the electron's group velocity is very small at the bottom state of the second miniband. Therefore these electrons have less contribution to the long wavelength photoresponse and this can also explain why responsivity at long wavelength range in 3-period superlattice is higher than in 15-period one and the few period superlattice can be operated at lower bias.

Because the transit distance of few-period superlattice may be short, the absorption coefficient can not be measured in our experiments. Because of the better absorption coefficient, photoresponse at short wavelength range of many-period superlattice may be better. Hence the period number of superlattice is a factor we have to tune for the optimum performance. Although the low absorption coefficient is a drawback for few periods superlattice, we still consider the few period superlattice is a better structure for SLIP because of its high responsivity, detectivity and operational temperature at long wavelength range.

\section{Conclusion}

We have compared the experimental results of SLIPs with different period number. By changing the polarity of bias, the different SLIP in one sample can be operated. The current blocking layer in SLIP structure can act as an energy filter and make the photoresponse tunable. Few-period superlattices have characteristics such as higher group velocity, less relaxation effect but lower absorption coefficient. For sample A, although the period numbers of the two superlattices are so close that the $I-V$ characteristics are almost the same, a better responsivity of the 3-period one than that of the 5-period one can still be identified. In sample B, because of the higher group velocity, we find better electrical and optical properties in the 3-period superlattice than in the 15-period one. Based on our experimental results, it is concluded that a superlattice with few periods has better responsivity, detectivity and higher operational temperature.

\section{Acknowledgements}

This work was supported by National Science Council of the Republic of China under contract number NSC 90-2215-E-002-015.

\section{References}

[1] B.K. Janousek, M.J. Daugherty, W.L. Bioss, M.L. Rosenbluth, M.J. O'Loughlin, H. Kanter, F.J. De Luccia, L.E. Perry, J. Appl. Phys. 67 (1990) 7068.

[2] A. Kastalsky, T. Duffield, S.J. Allen, J. Harbison, Photovoltaic detection of infrared light in a GaAs/AlGaAs superlattice, Appl. Phys. Lett. 52 (1988) 1320.

[3] O. Byungsung, J.-W. Choe, M.H. Francombe, K.M.S.V. Bandara, D.D. Coon, Long-wavelength infrared detection in a Kastalsky-type superlattice structure, Appl. Phys. Lett. 57 (1990) 503. 
[4] S.D. Gunapala, B.F. Levine, N. Chand, Bound to continuum superlattice miniband long wavelength GaAs/ $\mathrm{Al}_{x} \mathrm{Ga}_{1-x}$ As photoconductors, J. Appl. Phys. 70 (1991) 305.

[5] K.M.S.V. Bandara, J.-W. Choe, M.H. Francombe, GaAs/ AlGaAs superlattice miniband detector with $14.5 \mu \mathrm{m}$ peak response, Appl. Phys. Lett. 60 (1992) 3022.

[6] C.C. Chen, H.C. Chen, C.H. Kuan, S.D. Lin, C.P. Lee, Appl. Phys. Lett. 80 (2002) 2251.

[7] M. Helm, Semicond. Sci. Technol. 10 (1995) 557.
[8] C.C. Chen, H.C. Chen, M.C. Hsu, W.H. Hsieh, C.H. Kuan, S.Y. Wang, C.P. Lee, J. Appl. Phys. 91 (2002) 943.

[9] H.C. Liu, F. Capasso, Quantum well infrared photodetector physics and novel devices, in: Intersubband Trasitions in Quantum Wells-Physics and Device Applications I, Academic Press, London, 2000, pp. 135-136.

[10] T. Asano, S. Noda, K. Tomoda, Appl. Phys. Lett. 74 (1999) 1418. 